Zusammenfassend kann festgestellt werden, daß sich das beschriebene Kryoglobulin kaum von einem normalen $7 \mathrm{~S}$ Immunglobulin unterscheidet. In der Immunoelektrophorese bildet es eine bedeutend kürzere Präzipitationslinie, was auf Einheitlichkeit schließen läßt. Sein Molekulargewicht ist etwas höher als das der normalen $7 \mathrm{~S}$ Immunglobuline. Diese Differenz dürfte auf kleinen Mengen von Polymeren beruhen, die im Sedimentationsversuch nur zu einer Verschiebung der Werte und nicht zur Ausbildung eines eigenen Gradienten führen.
Der einzige augenscheinliche Unterschied war bei der Untersuchung der antigenwirksamen Stellen der Bruchstücke zu erkennen: während beim normalen $\gamma$-Globulin die H-Kette als Antigenträger wirksamer ist, findet man beim Kryoprotein stärkere Wirksamkeit in der II-Kette, die der L-Kette entspricht. $\mathrm{Da}$ die Stärkegelelektrophorese der Bruchstücke des Kryoproteins keine Einheitlichkeit ergibt, mag vielleicht daran liegen, daß die Bruchstücke zur Aggregation neigen.

Dem Österreichischen Forschungstat, der uns eine analytische Ultrazentrifuge zur Verfügung gestellt hat, sei auf diesem Wege aufrichtiger Dank ausgesprochen.

\title{
Literatur
}

1. Lerner, A. B. und G. R. Greenberg, J. biol. Chemistry 162, 429 (1946). - 2. Abrams, A., P. P. Cohen und O. O. Meyer, J. biol. Chemistry 181, 237 (1949). - 3. Barr, D. P., G. G. ReAder und C. H. W HeELeR, Ann. Int. Med. 32, 6 (1950). - 4. MANDEMA, E. und P. C. SchaAf, J. Laborat. Clin. Med. S. Louis 45, 261 (1955). - 5. Mackay, J. R., N. ERIKSEN und N. G. Motulsky, Amer. J. Med. XX, 564 (1956). - 6. BonsDORF, B. v., H. GROTH und T. PACKalen, Folia hämat. 59, 184 (1938). - 7. Holmberg, G. und A. GröNwall, Hoppe-Seyler's Z. physiol. Chem. 273, 199 (1942). -8. Blades, A. N., Brit. Med. J.4699, 169 (1951). -9.
Olhagen, B., Acta med. Scand. 138, 86 (1956). - 10. Caputo. A. und E. Appelta, Arch. Biochem. Biophysics 91, 201 (1960). - 11. GraBer, P. und P. Bưriti, Immunoelektrische Analyse, Elsevier Publishing Company, Amsterdam, New York (1964). - 12. Ouchterlony, Ö., Progr. Allergy 5, 1 (1958). - 13. Svedberg, T. und K. O. Pedersen, The Ultrazentrifuge, Oxford at the Clarendon Press (1940). - 14. FleischmanN, J. B., R. H. Pain und R. R. Porter, Arch. Biochem. Biophysics Suppl. 1, 174 (1962). - 15. Edelman, G. M. and M. D. Poulik, J. Exper. Med. 113, 861 (1961).
Dr. W. Palm

Universitätsplatz 2

A $8010 \mathrm{Graz}$

\section{Die gleichzeitige Bestimmung der Sekretionsrate von Cortisol und Corticosteron beim Menschen}

\author{
Von L. RaIth und H. J. KarL \\ Aus der I. Medizinischen Klinik der Universität München (Direktor: Prof. Dr. H. Scbwiegk)
}

(Eingegangen am 6. November 1967)

\begin{abstract}
Nach dem Isotopenverdünnungsprinzip wurden nach i. v. Injektion von $2 \mu \mathrm{C}{ }^{3} \mathrm{H}$-Cortisol und $0,2 \mu \mathrm{C}{ }^{14} \mathrm{C}$-Corticosteron gleichzeitig die Sekretionsraten dieser beiden Steroide beim Menschen bestimmt. Tetrahydrocortisol, ein Metabolit von Cortisol und Tetrahydrocorticosteron, ein Metabolit von Corticosteron wurden aus Urin nach Hydrolyse mit. $\beta$-Glucuronidase, Extraktion mit Methylenchlorid und Papierchromatographie in den Systemen Bush C, B/50 und LT 21/85 separiert. Nach säulenchromatographischer Reinigung der Papiereluate wurden Tetrahydrocortisol und Tetrahydrocorticosteron quantitativ mit Tetrazoliumblau-Reagenz bestimmt, die ${ }^{3} \mathrm{H}$ - und ${ }^{14} \mathrm{C}$ Impulse dieser Metaboliten gezählt und aus deren spezifischer Aktivität die Sekretionstaten von Cortisol und Corticosteron berechnet. Bei 12 Gesunden betrug die Cortisolsekretion $10,5 \mathrm{mg}$ bis $20,0 \mathrm{mg} /$ Tag und die Corticosteronsekretion 2,3 mg bis $4,2 \mathrm{mg} / \mathrm{Tag}$.
\end{abstract}

After the i. v. application of $2,0 \mu \mathrm{C}{ }^{3} \mathrm{H}$-cortisol and $0,2 \mu \mathrm{C}{ }^{14} \mathrm{C}$-corticosterone the secretion rates of these steroids were measured with aid of the isotope dilution technique. Tetrahydrocortisol, a metabolite of cortisol, and tetrahydrocorticosterone, a metabolite of corticosterone, were separated from urine after hydrolysis with $\beta$-glucuronidase, extraction with methylene chloride and paper chromatography in the systems BusH $\mathrm{C}, \mathrm{B} / 50$ and LT 21/85. The hormones were eluted from the paper and further purified by column chromatography. Tetrahydrocortisol and tetrahydrocorticosterone were then estimated quantitatively with tetrazolium blue reagent and the ${ }^{3} \mathrm{H}$-and ${ }^{14} \mathrm{C}$ impulses of the metabolites were counted. From the specific activity of tetrahydrocortisol and tetrahydrocorticosterone the sectetion rates of cortisol and corticosterone were calculated. In 12 normal persons the secretion rate of cortisol was $10,5 \mathrm{mg}-20,0 \mathrm{mg} / \mathrm{day}$ and the secretion rate of corticosterone $2,3 \mathrm{mg}-4,2 \mathrm{mg} /$ day.

Die Sekretionsrate eines Nebennierenrindenhormons kann nach dem Isotopenverdünnungsprinzip bestimmt werden (1,2). Bei dieser Methode wird nach Applikation eines radioaktiv markierten Corticosteroids die von der Nebennierentinde in der Zeiteinheit sezernierte bzw. die vom Organismus metabolisierte Hormonmenge aus der spezifischen Aktivität eines aus dem
Ưrin isolierten Metaboliten dieses Steroids berechnet. Unter den Bedingungen, die von Laumas und TarT $(3,4)$ ausführlich diskutiert wurden, ist diese Methode zuverlässig und genau. Sie hat sich bei der Bestimmung der Sekretionsrate von Cortisol $(1,5)$, von Corticosteron $(6,7)$ und von Aldosteron $(2,8)$ bewährt. Frood und Mitarbeiter (2) haben erstmals 
gleichzeitig die Sekretionstaten von zwei Steroiden, von Cortisol und von Aldosteron, mit ${ }^{14} \mathrm{C}$ oder ${ }^{3} \mathrm{H}$ markierten Hormonen untersucht.

Wir beschreiben eine Methode, mit der es möglich ist, die Sekretionsrate von Cortisol und von Corticosteron gleichzeitig zu bestimmen. Damit werden aus dem von der Nebennierenrinde sezernierten „Hormon-

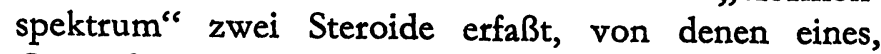
Cortisol, vorwiegend Glucocorticoidwirkung hat, während das andere, Corticosteron, den Kohlenhydratstoffwechsel und den Elektrolythaushalt gleichermaßen beeinflußt.

In dieser Arbeit wird auch auf die besonderen Probleme bei der gleichzeitigen Bestimmung von 2 Sekretionsraten mit verschieden radioaktiv markierten Steroiden eingegangen. Außerdem werden Ergebnisse bei Normalpersonen mitgeteilt.

\section{Methodik}

Reinsubstanzen und Reagenzien

$\left[1,2-{ }^{3} \mathrm{H}\right]$-Cortisol $=\left[1,2-{ }^{3} \mathrm{H}\right]-11 \beta, 17 \alpha, 21$-trihydroxy- $\Delta^{4}$ pregnen-3,20dion; spez. Akt.: 13,0 C/mMol (New England Nuclear Corporation, Bethesda).

$\left[4^{14} \mathrm{C}\right]$-Corticosteron $=\left[4^{14} \mathrm{C}\right]-11 \beta, 21-$ dihydroxy- $\Delta^{4}$ pregnen-3,20dion; spez. Akt.: $46 \mathrm{mC} / \mathrm{mMol}$ (New England Nuclear Corporation, Bethesda).

Cortison $=17 \alpha, 21$-dihydroxy- $\Delta^{4}$ pregnen-3,11,20-trion (Schering, Berlin).

11-Desoxycortisol (Reichsteins Substanz S) $=17 \alpha, 21$-dihydroxy$\Delta^{4}$ pregnen-3,20-dion (Schering, Berlin).

Tetrahydrocortisol(THF $=3 \alpha, 11 \beta, 17 \alpha, 21$-tetrahydroxypregnan20-dion (Steroid Reference Collection, London).

Tetrahydrocortison(THE) $=3 \alpha, 17 \beta, 21$-trihydroxy-pregnan-11,20dion (Steroid Reference Collection, London).

Tetrahydrocorticosteron(THB) $=3 \alpha, 11 \beta, 21$-trihydroxy-pregnan20-on (Steroid Reference Collection, London).

Silicagel, grade 923, mesh size 100-200 (W. R. Grace Davison Chemical Division, USA).

Tetrazoliumblau $=$ Blue Tetrazolium (Nutritional Biochemicals Corporation, Cleveland, USA).

PPO = 2,5-Diphenyloxazol; POPOP $=1,4$-bis-2-(5-phenyloxazolyl) benzol (Packard Instrument Company, Illinois, USA).

$\beta$-Glucuronidase, 375 Fishman-Einheiten/mg (Schering, Berlin).

Alle übrigen Reagenzien waren „pro analysi“ und/oder „,zur Chromatographie" von der Firma Merck AG, Darmstadt.

Der Reinheitsgrad der beiden radioaktiven Corticosteroide wurde papierchromatographisch in den beiden von Busir (9) angegebenen Systemen B/50 und LT 21/85 geprüft; er betrug über $95 \%$.

Verabreichung der radioaktiven Șteroide

$2,0 \mu \mathrm{C}{ }^{3} \mathrm{H}$-Cortisol und $0,2 \mu \mathrm{C}{ }^{14} \mathrm{C}$-Corticosteron, gelöst in absol. Âthanol $(0,5 \mathrm{ml})$, werden mit $20 \mathrm{~m} l$ physiol. NaCl-Lösung verdünnt und in 5 Min. i. v. injiziert.

\section{Messung der ${ }^{14} \mathrm{C}-\mathrm{Aktivität} \mathrm{im} \mathrm{Urin}$}

Die Aktivität des in 2 Portionen gesammelten Ürins (von 0-24 Stdn. p. i. und von $24-48$ Stdn. p. i.) wird nach der von FLood und Mitarbeitern (2) angegebenen Methode gemessen. Dabei wird $1 / 50$ der Urinmenge mit Wasser auf $50 \mathrm{ml}$ aufgefüllt und $0,6 \mathrm{ml}$ des verdünnten Utins mit $6,0 \mathrm{~m} l$ Äthanol und $10 \mathrm{~m} l$ Szintillatorflüssigkeit $\left(4,0 \mathrm{~g} \mathrm{PPO}^{1}\right)+0,1 \mathrm{~g}$ POPOP in $1000 \mathrm{ml}$ Toluol) versetzt. Die ${ }^{14} \mathrm{C}$-Impulse der Proben werden mit dem

1) Ablürrungen: $\mathrm{PPO}=2,5-\mathrm{Diphenyloxazol;} \mathrm{POPOP}=1,4$ bis-2(5-phenyloxazoly1)benzol, FE = Fishman-Einheiten, THF $=$ Tetrahydrocortisol, THE $=$ Tetrahydrocortison, $\mathrm{THB}=$ Tetrahydrocorticosteron, 17-OHCS = 17-Hydroxycorticosteroide.
Flüssigkeitsszintillationsspektrometer Packard Tri-Carb Modell 3002 bei folgender Einstellung 2mal 100 Min. gezählt: Diskriminator $A=50$, Diskriminator $B=500$, gain $=3$. Das , quenching“ der Proben wird mit einem internen Standard bestimmt, dann die ${ }^{14} \mathrm{C}$-A ktivität der gesamten Urinmenge berechnet und in Prozent der injizierten Aktivität von ${ }^{14} \mathrm{C}$-Corticosteron umgerechnet.

Separierung des Cortisolmetaboliten Tetrahydrocortisol und des Corticosteronmetaboliten Tetrahydrocorticosteron

Ausgangsmaterial: Tetrahydrocortisol (THF) und Tetrahydrocorticosteron (THB) werden aus $1 / 10$ der Urinmenge $0-24$ Stdn. p. i. separiert; sind im Urin 24-48 Stdn. p. i. mehr als 5\% der injizierten Aktivität von ${ }^{14} \mathrm{C}$-Corticosteron vorhanden, wird $1 / 10$ Vol. der Urinmenge $0-48 \mathrm{Stdn}$. p. i. verwendet.

Hydrolyse: Der Urin wird mit $1 / 10$ Vol. Natriumacetat $(0,2 \mathrm{M})$ und $1 / 10$ Vol. Essigsäure $(0,2 \mathrm{M})$ versetzt und mit $300 \mathrm{FE} \beta$-Glucuronidase pro $\mathrm{m} /$ Urin bei $\mathrm{pH} 4,6$ und $45^{\circ} 24 \mathrm{Stdn}$. hydrolysiert.

Extraktion und Reinigung: Der hydrolysierte Urin wird 3mal mit Methylenchlorid (V/V) extrahiert und die vereinigten Extrakte $2 \mathrm{mal} \mathrm{mit} 1 / 10$ Vol. $0,1 \mathrm{~N} \mathrm{NaOH}$ sowie $2 \mathrm{mal} \mathrm{mit} 1 / 10$ Vol. Wasser gewaschen und anschließend mit Natriumsulfat getrocknet. Natronlauge und Waschwasser werden mit Methylenchlorid (V/V) rückextrahiert und die vereinigten Extrakte unter reduziertem Druck abgedampft. Anschließend wird dieser vorgereinigte Extrakt nach Petrerson (10) zwischen Petroläther und wäßr. Äthanol (Petroläther (Kp 60-80 $) /$ Äthanol/Wasser $=100: 10: 30$ $\mathrm{V} / \mathrm{V})$ verteilt und die Steroide aus der wäßr. Phase mit Methylenchlorid (3mal 2 Vol.) rückextrahiert. Die Extrakte werden vereinigt und bei $40^{\circ}$ unter vermindertem Druck abgedampft.

Papierchromatograpbie: Im ersten Chromatogramm, System Bush C (Toluol/Äthylacetat/Methanol/Wasser $=9: 1: 5: 5 \mathrm{~V} / \mathrm{V}$ ), werden die weniger polaren Corticosteronmetaboliten von den höher polaren Cortisolmetaboliten getrennt. Der Extrakt wird auf Whatmanpapier Nr. 1 (Breite der Streifen $8 \mathrm{~cm}$, Länge $33 \mathrm{~cm}$ ) absteigend nach einer Äquilibrierdauer von 60 Min. bei $23^{\circ}$ chromatographiert; Laufzeit etwa $3 \mathrm{Stdn}$. Auf Parallelstreifen laufen als Leitsubstanzen Cortison und Reichsteins Substanz S, die mit Tetrazoliumblau-Reagenz (1 Vol. 1proz. wäßr. Lösung von Tetrazoliumblau +9 Vol. $2 \mathrm{~N} \mathrm{NaOH}$ ) sichtbar gemacht werden. Das Chromatogramm wird dann in 2 Zonen geteilt.

Zone I: $2 \mathrm{~cm}$ unterhalb des Starts bis zum Beginn der Cortisonzone auf dem Parallelstreifen $\left(R_{\mathrm{F}}=0,40\right)$.

Zone II: Vom Ende der Zone I bis zum Ende der Zone von Reichsteins Substanz S auf dem Parallelstreifen $\left(R_{F}=0,9\right)$.

Beide Zonen werden $3 \mathrm{mal} \mathrm{mit} 50 \mathrm{~m} l$ Methanol eluiert, die Eluate jeweils halbiert und zur Trockene eingeengt.

Die beiden Extrakthälften der Zone I, die unter anderem THF enthalten, werden auf 2 parallelen Chromatographiestreifen (Wathman Nr. 1, Breite $3 \mathrm{~cm}$, Länge $40 \mathrm{~cm}$ ) am Start aufgetragen und im System Bush B/50 (Benzol/Methanol/Wasser = 10:5:5 V/V) absteigend rechromatographiert (Äquilibrierdauer $60 \mathrm{Min}$., Laufzeit 24 Stdn. bei $2^{\circ}$ ); auf Parallelstreifen laufen die Reinsubstanzen THF und THE. Die beiden Hälften der Eluate von Zone II, die unter anderem THB enthalten, werden unter analogen Bedingungen 28 Stdn. im System Busk LT 21/85 (Petroläther ( $\mathrm{K}_{\mathrm{P}}$ $60-80^{\circ}$ )/Toluol/Methanol/Wasser $=66,6: 33,3: 85: 15 \mathrm{~V} / \mathrm{V}$ ) rechromatographiert. Auf einem Parallelstreifen läuft als Leitsubstanz THB.

Von jedem Chromatogramm wird jeweils ein Streifen mit der Hälfte des Extrakts mit Tetrazoliumblau-Reagenz angefärbt und die THF und THB entsprechende Zone der Parallelstreifen (mit der zweiten Hälfte des Extrakts) $3 \mathrm{mal}$ mit $5 \mathrm{~m} /$ Methanol eluiert und die beiden Eluate bei $40^{\circ}$ unter vermindertem Druck abgedampft.

Säulenchromatographie: 2 Säulen mit je $2 \mathrm{~g}$ Silicagel (Säulendurchmesser $10 \mathrm{~mm}$ ) werden mit $15 \mathrm{~m} /$ Methanol/Chloroform (V/V) vorgewaschen, die beiden Eluate von den Papierchromatogrammen mit THF und THB quantitativ übertragen und die Steroide 
mit $20 \mathrm{~m} /$ Methanol/Chloroform (V/V) eluiert. Nach Abdampfen der Eluate wird der Trockenrückstand mit THF und THB in $3 \mathrm{~m} /$ absol. Äthanol gelöst. $2 \mathrm{ml}$ dienen zur quantitativen Bestimmung und $1 \mathrm{~m} /$ zur Messung der Radioaktivität.

Kolorimetrische Bestimunungen von THF und THB: Die beiden Metaboliten THF und THB werden nach SEEBER (11) quantitativ bestimmt: $2 \mathrm{~m} l$ des in $3 \mathrm{~m} l$ absol. Äthanol gelösten Eluats aus der Silicagelsäule mit THF oder THB werden mit $0,2 \mathrm{ml}$ Tetrazoliumblau-Reagenz (15 mg Tetrazoliumblau gelöst in $3 \mathrm{ml}$ absol. Athanol) und $0,2 \mathrm{~m} l$ einer alkohol. Tetramethylammoniumhydroxydlösung $(0,5 \mathrm{~m} / 10$ proz. Tetramethylammoniumhydroxyd $+4,5 \mathrm{~m} /$ absol. Äthanol) versetzt. Nach $25 \mathrm{Min}$. im Thermostat bei $25^{\circ}$ werden $0,05 \mathrm{~m} /$ Eisessig zugegeben und die Extinktion der Probe mit dem Spektralphotometer (Beckman DU) bei $510 \mathrm{~nm}$ (Schichtdicke $10 \mathrm{~mm}$ ) gegen den Leerwert der Reagenzien gemessen. Nach Abzug des Papierleerwertes $(<5 \%$ der Extinktion des Hormonwertes) wird die Steroidmenge der Proben an einer Eichkurve, die mit den beiden Reinsubstanzen THF und THB aufgestellt wird, abgelesen und in mg pro Urintagesmenge umgerechnet. Bei jedem Ansatz werden zur Kontrolle der Eichwerte $10 \mu \mathrm{g}$ THF und THB mitbestimmt.

Messung der Aktivität von ${ }^{3} H-T H F$ und ${ }^{14} C-T H B$ : Der Rest der Eluate aus den beiden Silicagelsäulen (je $1 \mathrm{~m} l$ ) wird quantitativ in Meßgläschen übertragen und das Lösungsmittel abgedampft. Nach Zugabe von $1 \mathrm{ml}$ absol. Äthanol und $10 \mathrm{ml}$ Szintillatorflüssigkeit wird die ${ }^{14} \mathrm{C}$ - oder ${ }^{3} \mathrm{H}$-Aktivität der Proben mit dem Flüssigkeitsszintillationsspektrometer nach der Ausblendmethode von OkITA und Mitarbeitern (15) gemessen (Kanal I - Diskriminator $A=50$, Diskriminator $B=500$; gain $=2$; Kanal II Diskriminator $C=50$, Diskriminator $D=1000$; gain $=100$ ).

Berechnung der Sekretionsrate: Die Sekretionsraten wurden nach der vereinfachten Formel von Pearladan (12) und Peterson und Pierce (13) berechnet (7).

$$
\begin{gathered}
\text { Sekretionsrate von Cortisol } \\
(\mathrm{mg} / \mathrm{Tag})
\end{gathered}=\frac{\mathrm{J}_{\mathrm{i}} \cdot \mathrm{X}}{\mathrm{J}_{\mathrm{x}} \cdot \mathrm{t}}
$$

$\mathrm{J}_{\mathrm{i}}=$ Aktivität (Imp./Min.) des injizierten ${ }^{3} \mathrm{H}$-Cortisol gezählt in Kanal II

$\mathrm{J}_{\mathrm{x}}=$ Aktivität (Imp./Min.) von ${ }^{3} \mathrm{H}$-THF gezählt in Kanal II umgerechnet auf die gesamte Urinmenge. Dabei gilt:

$\mathrm{J}_{\mathrm{X}}=(\mathrm{Imp} . / \mathrm{Min}$. in Kanal II - c $\cdot$ Imp./Min. in Kanal I) $\cdot 60$

$\mathrm{c}=$ Verhältnis der ${ }^{14} \mathrm{C}$-Impulse in Kanal II/Kanal I

$\mathrm{X}=\mathrm{mg}$ THF umgerechnet auf die gesamte Urinmenge

$\mathrm{t}=$ Urinsammelperiode in Tagen

$$
\underset{(\mathrm{mg} / \mathrm{Tag})}{\operatorname{Sekretionsrate} \text { von Corticosteron }}=\frac{\mathrm{J}_{\mathrm{i}} \cdot \mathrm{X}}{\mathrm{J}_{\mathrm{x}} \cdot \mathrm{t}}
$$

$\mathrm{J}_{\mathbf{i}}=$ Aktivität (Imp./Min.) des injizierten ${ }^{14} \mathrm{C}$-Corticosteron gezählt in Kanal I

$\mathrm{J}_{\mathrm{x}}=$ Aktivität (Imp./Min.) von ${ }^{14} \mathrm{C}-\mathrm{THB}$ gezählt in Kanal I

$\mathrm{X}=\mathrm{mg}$ THB umgerechnet auf die gesamte Urinmenge

$\mathbf{t}=$ Urinsammelperiode in Tagen

\section{Ergebnisse und Diskussion}

Die Applikation des radioaktiven Steroids zur Bestimmung einer Sekretionsrate nach dem İsotopenverdünnungsprinzip erfolgt intravenös, da nicht geklärt ist, ob nach oraler Verabreichung die einzelnen Steroide von allen Probanden unverändert und vollkommen resorbiert werden und ob die aus dem Verdauungstrakt aufgenommenen Hormone ebenso metabolisiert werden wie die von der Nebennierentinde sezernierten Steroide. Wir injizierten die in Äthanol gelösten radioaktiven Steroide verdünnt mit $20 \mathrm{ml}$ physiol. NaCl-Lösung. Der Verlust, der bei der In- jektion entsteht, ist gering. Im Mittel verblieben nur $0,39 \%(0,04 \%$ bis $0,79 \% ; n=8)$ der Aktivität in der Kanüle und in der Injektionsspritze.

Transport und Stoffwechsel von endogenem Cortisol und Corticosteron werden durch die zugeführten Isotope nicht beeinflußt, denn die von uns injizierten Hormonmengen $(5,6 \mu \mathrm{g}$ Cortisol und $1,5 \mu \mathrm{g}$ Corticosteron) liegen unter $1 \%$ des Hormonpools dieser beiden Steroide, den Peterson (14) für Cortisol mit 1,2 bis $2,4 \mathrm{mg}$ und für Corticosteron mit 0,2 bis $0,4 \mathrm{mg}$ angibt.

Die ${ }^{3} \mathrm{H}$ - und ${ }^{14} \mathrm{C}$-Aktivität des Ưrins kann nach gleichzeitiger Injektion von ${ }^{3} \mathrm{H}$-Cortisol und ${ }^{14} \mathrm{C}$-Corticosteron mit der sogenannten Ausblendmethode (15) bestimmt werden. Wir fanden dabei den Standardmeßfehler für die Zählung der ${ }^{14} \mathrm{C}$-Aktivität des Úrins mit einem Variabilitätskoeffizienten von 1,2\% relativ gering. Dagegen ist die Bestimmung der Tritiumaktivität des Harns mit dieser Zählmethode bei den geringen Impulsraten und bei dem "quenching “ der Proben mit einem größeren Meßfehler behaftet. Nachdem der Ausscheidungsmodus für Cortisol und Corticosteron annähernd gleich ist (10) haben wir, um uns über die Ausscheidung der Radioaktivität im Urin zu orientieren, nur die ${ }^{14} \mathrm{C}$-Aktivität bestimmt. Im Mittel fanden wir in den ersten 24 Stdn. p. i. $83,3 \%$ der injizierten ${ }^{14} \mathrm{C}$-Aktivität im Urin $(n=13)$. Dieser Wert ist in der gleichen Größenordnung wie nach Injektion von ${ }^{14} \mathrm{C}$-Corticosteron allein $(6,7)$.

Im Extrakt nach Hydrolyse mit $\beta$-Glucuronidase betrug bei der Messung mit der Ausblendmethode (15) die ${ }^{3} \mathrm{H}$-Aktivität im Mittel $29,6 \%$ und die ${ }^{14} \mathrm{C}$-Aktivität im Mittel 30,7\% ( $\left.n=13\right)$, bezogen auf die jeweils injizierte Aktivität.

Der Verlust bei den einzelnen Arbeitsgängen zur Separierung-der Metaboliten von Cortisol und Corticosteron, THF und THB, geht aus Tabelle 1 hervor.

Tab. 1

Verlust bei den einzelnen Arbeitsgängen

\begin{tabular}{lcc}
\hline \multirow{2}{*}{$\quad$ Arbeitsgang } & \multicolumn{2}{c}{ Verlust von } \\
& $\begin{array}{c}{ }^{1 \triangleleft} \text { C-Aktivität } \\
\%\end{array}$ & $\begin{array}{c}{ }^{3} \mathrm{H}-\text { Aktivität } \\
\%\end{array}$ \\
\hline Extraktion & $5,0-7,8$ & $4,5-8,8$ \\
Petrolätherverteilung & $2,9-4,3$ & $4,2-6,0$ \\
Papierchromatographie & & \\
$\quad$ 1. Chromatogramm & $1,5-5,5$ & $2,0-6,0$ \\
2. Chromatogramm & $0,5-2,7$ & $3,0-7,2$ \\
Säulenchromatographie & $>0,5$ & $>0,5$ \\
\hline
\end{tabular}

Der Gesamtverlust beträgt $20 \%$ bis $25 \%$ ( $n=8)$. Das Endergebnis wird dadurch zwar nicht beeinflußt, da die Sekretionsrate der Steroide aus der spezifischen Aktivität der Steroidmetaboliten und nicht nur aus der Absolutmenge berechnet wird. Der Verlust sollte trotzdem möglichst geting sein, um bei der relativ kleinen Urin-Ausgangsmenge in den optimalen Bereich der Nachweisreaktion zu kommen.

Die Reinbeit von THF und THB wurde durch einen Vergleich der Spektralkurven mit den von reinem THF und THB in konzentrierter Schwefelsäure nach der Methode von Axelrod (16) geprüft. Die separierten 
Tab. 2

Gleichzeitig bestimmte Sekretionsraten von Cortisol und Corticosteron bei 12 Normalpersonen

\begin{tabular}{|c|c|c|c|c|c|c|c|c|c|c|}
\hline Name & $\begin{array}{r}\text { Alter und } \\
\text { Geschlecht }\end{array}$ & $\begin{array}{c}\text { 17-OHCS } \\
\text { im Urin } \\
\text { mg/24 Stdn. }\end{array}$ & $\begin{array}{c}\text { 1aC-Aktivi- } \\
\text { tät im Urin } \\
0-24 \text { Stdn. } \\
\text { p. i. } \\
\% \text { der inj. } \\
\text { 10C-Aktivität }\end{array}$ & $\begin{array}{c}\text { THF im } \\
\text { Urin } \\
\text { mg/24Stdn. }\end{array}$ & $\begin{array}{c}\text { Aktivität } \\
\text { von } \\
\text { 'H-THF } \\
\text { \% der inj. } \\
\text { 'H-Aktivität }\end{array}$ & $\begin{array}{l}\text { Sekretions- } \\
\text { rate von } \\
\text { Cortisol } \\
\text { mg/24 Stdn. }\end{array}$ & $\begin{array}{c}\text { THB im } \\
\text { Urin } \\
\text { mg/24 Stdn. }\end{array}$ & $\begin{array}{l}\text { Aktivität } \\
\text { von } \\
\text { 14C-THB } \\
\% \text { der inj. } \\
\text { }{ }^{4} \text { C-Aktivität }\end{array}$ & $\begin{array}{l}\text { Sekretions- } \\
\text { rate von } \\
\text { Cortico- } \\
\text { steron } \\
\text { mg/24 Stdn. }\end{array}$ & $\begin{array}{c}\text { Quotient } \\
\text { der Sekre- } \\
\text { tionsraten } \\
\text { Cortisol/ } \\
\text { Cortico- } \\
\text { steron }\end{array}$ \\
\hline
\end{tabular}

\begin{tabular}{|c|c|}
\hline $\begin{array}{l}\text { R. G. } \\
+\end{array}$ & $24 \%$ \\
\hline $\begin{array}{l}\text { B. J. } \\
\text { V. H. } \\
\text { T. P. } \\
\text { M. M. } \\
\text { S. E. } \\
\text { S. W. } \\
\text { S. K. } \\
\text { G. G. } \\
\text { H. J. } \\
\text { B. F. } \\
\text { U. B. }\end{array}$ & 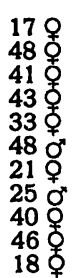 \\
\hline
\end{tabular}

\begin{tabular}{lll}
$\mathbf{4}, 7$ & $\mathbf{8 0 , 1}$ & 0 \\
$\mathbf{3}, \mathbf{8}$ & $\mathbf{7 5 , 5}$ & \\
$\mathbf{3}, 5$ & $\mathbf{7 2 , 7}$ & 0 \\
$\mathbf{3}, \mathbf{2}$ & $\mathbf{8 8 , 0}$ & 0 \\
$\mathbf{3}, 4$ & $\mathbf{7 1 , 4}$ & 0 \\
$\mathbf{2}, \mathbf{8}$ & $\mathbf{6 8 , 2}$ & 0 \\
$\mathbf{4 , 4}$ & $\mathbf{9 0 , 3}$ & 0 \\
$\mathbf{4}, 0$ & $\mathbf{8 1 , 3}$ & 0 \\
$\mathbf{2}, \mathbf{5}$ & 90,0 & 0 \\
$\mathbf{4}, 6$ & $\mathbf{8 1 , 0}$ & 0 \\
$\mathbf{5}, 0$ & $\mathbf{8 9}, 6$ & 0 \\
$\mathbf{2}, 1$ & $\mathbf{8 3}, 6$ & 0 \\
$\mathbf{2 , 1}$ & 96,6 & 0 \\
\hline
\end{tabular}

0,59
0,60
0,20
0,65
0,45
0,29
0,62
0,38
0,44
0,26
0,25
0,26
0,30

\begin{tabular}{|c|c|}
\hline 4,0 & 14,7 \\
\hline $\begin{array}{l}3,0 \\
1,6 \\
3,8 \\
4,3 \\
2,0 \\
3,5 \\
2,3 \\
3,2 \\
1,6 \\
2,0 \\
2,0 \\
2,2\end{array}$ & $\begin{array}{l}20,0 \\
12,5 \\
17,1 \\
10,5 \\
14,5 \\
17,7 \\
16,5 \\
13,7 \\
16,2 \\
12,5 \\
13,0 \\
13,6\end{array}$ \\
\hline
\end{tabular}

$\overline{0,17}$
0,20
0,17
0,15
0,19
0,14
0,30
0,18
0,19
0,10
0,12
0,15
0,18

$\overline{6,0}$
5,2
6,4
4,3
8,4
5,8
7,9
4,8
4,7
3,0
4,3
3,6
4,5

Steroide hatten die gleichen Extinktionsmaxima wie die reinen Hormone (THF bei $510 \mathrm{~nm}, 420 \mathrm{~nm}$ und $330 \mathrm{~nm}$; THB bei 440 bis $430 \mathrm{~nm}$ und $320 \mathrm{~nm}$ ).

Auf den Chromatogrammen waren im System LT 21/85 in der Zone von THB $+{ }^{14} \mathrm{C}$-THB unter $0,1 \%$ der injizierten Tritium-Aktivität nachweisbar und im System B/50 in der Zone von THF + ${ }^{3} \mathrm{H}$-THF weniger als $0,2 \%$ der applizierten ${ }^{14} \mathrm{C}$-Aktivität. Trotz dieser nur geringen Überlagerung der ${ }^{14} \mathrm{C}$ - und ${ }^{3} \mathrm{H}$-Aktivitäten wurden die Impulse mit der Ausblendmethode gezählt. Ein "quenching" der Proben war nicht nachweisbar.

Genauigkeit und Empfindlichkeit der gesamten Methode hängen von der Genauigkeit und Empfindlichkeit der Tetrazoliumblau-Reaktion und der Genauigkeit der Zählung der ${ }^{14} \mathrm{C}$ - und ${ }^{3} \mathrm{H}$-Impulse der Steroidmetaboliten ab. - Die Fehlerbreite bei der quantitativen Bestimmung von Steroiden mit der TetrazoliumblauReaktion ist mit ungereinigten Eluaten von Papierchromatogrammen groß, da die Papierleerwerte hohe und vor allem unterschiedliche Extinktionen ergeben. Erst nach Reinigung der Eluate auf einer Silicagelsäule betrug die Extinktion der Papierleerwerte nur noch maximal 0,01. Bei der quantitativen Bestimmung von $10 \mu \mathrm{g}$ reinem THF oder THB betrug die Standard- abweichung $s=0,1$, die Wiederfindung nach einmaliger Papier- und Säulenchromatographie im Mittel $94,5 \%(92 \%$ bis $101 \%)(n=10)$. Die untere Nachweisgrenze mit der Tetrazoliumblau-Reaktion liegt bei $0,5 \mu \mathrm{g}$; bis $40 \mu \mathrm{g}$ folgt die Reaktion dem LAMBERTBeErschen Gesetz. - Bei der Zählung der Impulse von ${ }^{14} \mathrm{C}-\mathrm{THB}$ ergab sich ein Variabilitätskoeffizient im Mittel von $1,09 \%(0,73 \%$ bis $1,05 \%)$ und bei der Zählung der Impulse von ${ }^{3} \mathrm{H}-\mathrm{THF}$ im Mittel von $0,85 \%(0,73 \%$ bis $1,04 \%)(n=20)$.

In Tabelle 2 sind die Ergebnisse der gleichzeitig bestimmten Sekretionsraten von Cortisol und Corticosteron von 12 Normalpersonen (10 Frauen und 2 Männer) im Alter von 17 bis 48 Jahren zusammengestellt. Die Cortisolsekretion betrug 10,5 bis $20,0 \mathrm{mg}$ / 24 Stdn., die Corticosteronsekretion 2,3 bis $4,2 \mathrm{mg} /$ 24 Stdn. Im Mittel sind die Sekretionsraten von Cortisol $(14,8 \mathrm{mg} / 24 \mathrm{Stdn}$.) und Corticosteron $(3,3 \mathrm{mg} / 24 \mathrm{Stdn}$.) in der gleichen Größenordnung wie bei Bestimmung jeder Sekretionsrate einzeln bei Normalpersonen (17). Der Quotient der Sekretionsraten Cortisol/Corticosteron betrug im Mittel 4,4. Bei Einzelbestimmungen der Sekretionsraten an verschiedenen Tagen war nach vaN DER STRAETEN und Mitarbeitern (6) dieser Quotient mit 6,7 größer.

\section{Literatur}

1. Cope, C. L. und E. G. Brack, Clin. Sc. London 17, 147 (1958). 2. Flood, C., D. S. Layne, S. Ramcharan, E. Rossipal, J. F. TAIT und S. A. S. TAIT, Acta endocr. (K'hvn) 36, 237 (1961). 3. Laumas, K. R., J. F. Tart und S. A. S. Tart, Acta endocr. (K'hrn) 36, 265 (1961). - 4. TAIt, J. F., J. Clin. Endocr. Springfield 23, 1285 (1963). - 5. KARL, H. J., in: Radio-Isotope in der Endokrinologie S. 365 hrsg. von G. HofFManN, F. K. Schattauer, Stuttgart (1965). - 6. VAN DER STraeten, M., A. Vermeulen und N. OrIE, Acta endocr. (K'hrn) 43, 430 (1963). - 7. KarL, H. J. und L. RaIth, Klin. Wschr. 43, 863 (1965). - 8. SieG̈ENTHALER, W. E., A. J. Dowdy und J. A. Luetscher, J. Clin. Endocr. Springfield 22, 172 (1962). - 9. Busk, I. E., The chromatography of steroids. New York-Oxford-London-Paris: Pergamon Press (1961). - 10. Peterson, R. E., J. B. WygngaArden, S. L. Guerra, B. B. Brodie und J. J. Bunim, J. clin. Invest. 34, 1779 (1955). 11. SeEker, E., Naunyn-Schmiedebergs Arch. exper. Path. 242, 101 (1961). - 12. Pearlman, W. H., Ciba Found. Coll. on Endocr. 11, 233 (1957). - 13. Peterson, R. E. und C. E. Pierce, J. clin. Invest. 39, 741 (1960). - 14. Peterson, R. E., Recent Progr. Hormone Res. N. Y. 15, 231 (1959). - 15. OkrtA, G. T., J. J. Kabara, F. Richardson und G. V. Leroy, Nucleonics 15, 111 (1957). - 16. Axelrod, L. R., J. biol. Chemistry 205, 173 (1953). 17. KARL, H. J. und L. RaIth, Klin. Wschr. 43, 867 (1965).

Dr. L. Raith u. Priv.-Doz. Dr. H. J. Karl 8000 München 15

Ziemssenstr. 1 